\title{
EFFECTS OF ANNIHILATION ON RESONANCES IN THE NN SYSTEM
}

\author{
Yu. A. SIMONOV ${ }^{+}$and J. A. TJON \\ Institute for Theoretical Physics, University of Utrecht, Utrecht, The Netherlands
}

Received 16 June 1978

(Revised 9 October 1978)

\begin{abstract}
The widths and positions of resonances of the $\mathrm{N} \overline{\mathrm{N}}$ system for $l=0,1$ are studied in a relativistic potential model, where the absorption is described in terms of an annihilation box diagram. The trajectories of the singularities in the complex $E$-plane are determined as a function of the coupling strengths. For coupling constants fitted to the experimental cross sections, the widths of the quasinuclear states are of the order of $40 \mathrm{MeV}$. In addition, also resonances occur due to the coupling to the annihilation channels. These coupled channel resonances can have small widths and give in general rise to complicated Argand plots. For their description a simple phenomenological form for the scattering amplitude is used.
\end{abstract}

\section{Introduction}

The experimental discovery of some peaks in the $N \bar{N}$ system during the past few years ${ }^{1}$ ) has led to discussions about the possible interpretation of these peaks. Experimentally some of the bumps are narrow $[M, \Gamma: 1936,<8 ; 2020,24 ; 2204$, $16 \mathrm{MeV}$; see ref. $\left.\left.{ }^{1}\right)\right]$, others in the same mass region are wide $(2190,90 ; 2350,160 \mathrm{MeV})$. Theoretically from quark dual theory one would rather expect the baryonium peaks in the $\mathrm{N} \overline{\mathrm{N}}$ system to be narrow at least for some states ${ }^{2}$ ). However no reliable theoretical estimates are possible. In the potential type approach ${ }^{3}$ ) for the $N \bar{N}$ resonances, called quasi-nuclear states, the magnitude of the width of these resonances has been strongly debated ${ }^{4-9}$ ). One point of view, supported by Shapiro et al. ${ }^{3,4}$ ) suggests that the widths $\Gamma$ and energy shifts $\Delta E$ due to annihilation are small ( $\$ 10$ $\mathrm{MeV}$ ) even for s-states. However, no strong arguments and exact calculations support that point of view. On the other hand, calculations have been carried out in the optical model ${ }^{5,6}$ ) where annihilation is simulated by a purely imaginary optical potential and in a boundary condition model ${ }^{7}$ ). The conclusions reached in these models were that the annihilation strongly affects the bound states and resonances, obtained from the one boson exchange (OBE) forces. As a result the width becomes so large that the resonances do not show up in the cross sections. The results were obtained by examining the scattering amplitude at real energies using the Bryan-Phillips optical potential ${ }^{10}$ ) as input. More recently, to study the

\footnotetext{
+ Permanent address: ITEP, Moscow, USSR.
} 
actual positions of the bound state singularities for s-waves in the NN system the many channel $N / D$ method was used ${ }^{8}$ ) where the strength of annihilation was fitted to reproduce the experimentally known annihilation cross section $\sigma_{\text {inel }}$. In view of the possible drawbacks of the $N / D$ method such as the occurrence of ghosts and other effects due to the use of insufficient dynamical input, it is worthwhile to study the above questions in a different formalism.

In this paper we use the many-channel Logunov-Tavkhelidze-Blankenbecler-Sugar (LTBS) equations ${ }^{11}$ ) to describe the NN system and annihilation to different pionic channels. Disregarding the interaction between the pions we obtain a one channel equation with the OBE and annihilation box diagram, shown in fig. 1, as driving forces. This equation can be studied in the complex energy plane and in this way we can find the actual position of the poles as a function of the annihilation strength $\lambda_{3}$. For values of $\lambda_{3}$ fitted to experimental cross sections we find, that the width and energy shift are of the order of $40 \mathrm{MeV}$, also for the $l=1$ case. Similar as in ref. ${ }^{8}$ )

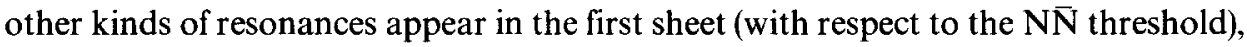
which we will call here "coupled channel resonances" or "CC poles", since they are produced by a strong coupling between any two (or more) channels. In our case, they arise from the coupling between the $\mathrm{N} \overline{\mathrm{N}}$ channel and all the pionic channels.
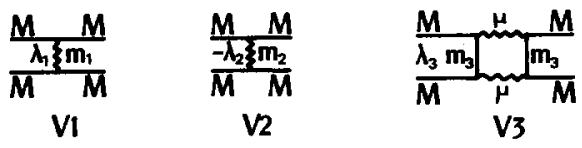

Fig. 1. The diagrams representing an attractive and repulsive Yukawa interaction $V_{1}$ and $V_{2}$ and the annihilation box diagram $V_{3}$.

For real energies we may represent the $\mathrm{N} \overline{\mathrm{N}}$ amplitudes with Argand plots. In so doing, we found that our $1=1$ loops are similar to those of Myhrer and Gersten ${ }^{5}$ ) obtained for the Bryan-Phillips optical potential. To find all the nearby poles, corresponding to our and Myhrer-Gersten Argand plots, we have used an elegant phenomenological description with formulae of the Dalitz-Tuan type ${ }^{12}$ ) and to our surprise have discovered three nearby poles in both cases (both of a quasi-nuclear and coupled channel type). Our results agree qualitatively with the point of view that the quasi-nuclear states can be moderate $(\Gamma \gtrsim 40 \mathrm{MeV}$ for $l=0,1)$. However from this study it follows that a deformed Argand plot indicates that the situation can be very complicated near $N \bar{N}$ threshold, rather than only the occurrence of a very distant pole as was assumed in refs. ${ }^{5,6}$ ).

The paper is organized as follows. In sect. 2 we describe the model used in our study. The unitarity condition and analytic properties of the $N \bar{N}$ amplitude are discussed in sect. 3. A simple and useful representation of this amplitude is introduced. In sect. 4 we present the results of the numerical calculations and discuss their physical implications. 


\section{The model}

Let us consider a two-channel situation, where channel one consists of two relativistic particles with equal mass $M$, while channel two has particles with mass $\mu$ with $M>\mu$. We assume that the number of particles in channel two is given by two. The more complicated situation with more than two particles is simulated in this model by introducing an appropriate phase space factor. In channel one the interaction consists of an attractive exchange of a particle with mass $m_{1}$ and a repulsive exchange of a particle with mass $m_{2}$. The coupling between the two channels is described by an exchange of a particle of mass $m_{3}$ while we assume that there is no direct interaction between the particles in channel two. As a consequence our two-channel problem reduces in an exact way to the one-channel relativistic equation of the LTBS type for the scattering matrix $t_{1}$ for channel one,

$$
t_{l}\left(p, p^{\prime} ; E\right)=V_{l}\left(p, p^{\prime} ; E\right)+\frac{1}{2} \pi \int_{0}^{\infty} \frac{\mathrm{d} p^{\prime \prime}}{\sqrt{p^{\prime \prime 2}+1}} \frac{V_{l}\left(p, p^{\prime \prime} ; E\right) t_{l}\left(p^{\prime \prime}, p^{\prime} ; E\right)}{p^{\prime \prime 2}-E-i \varepsilon}
$$

where the units are used $\hbar=M=c=1$ and the normalisation of $t_{l}$ is chosen in such a way that for on-shell values of the momentum $\hat{p}=\sqrt{ } E=\sqrt{\frac{1}{4} s-1}$ we have

$$
t_{l}\left(\hat{p}, \hat{p} ; \hat{p}^{2}\right)=\left(\frac{2}{\pi}\right)^{2} \hat{p}\left(\hat{p}^{2}+1\right)^{\frac{1}{2}} \frac{\eta \mathrm{e}^{2 i \delta}-1}{2 i} .
$$

The effective potential $V_{l}$ consists of three parts

$$
V_{l}=V_{l}^{(1)}+V_{l}^{(2)}+V_{l}^{(3)} \equiv V_{\mathrm{s}}+V_{3}
$$

with

$$
\begin{gathered}
V_{\mathrm{s}}=\lambda_{1} Q_{l}\left(\frac{p^{2}+p^{\prime 2}+m_{1}^{2}}{2 p p^{\prime}}\right)-\lambda_{2} Q_{l}\left(\frac{p^{2}+p^{\prime 2}+m_{2}^{2}}{2 p p^{\prime}}\right) \\
V_{3}=\lambda_{3} \int_{0}^{\infty} \frac{\kappa(k) \mathrm{d} k}{k^{2}-q^{2}-i \varepsilon} Q_{l}\left(\frac{p^{2}+k^{2}+m_{3}^{2}}{2 p k}\right) Q_{l}\left(\frac{p^{\prime 2}+k^{2}+m_{3}^{2}}{2 p^{\prime} k}\right),
\end{gathered}
$$

where $q=\sqrt{E-E_{t}}$ with $E_{t}=\mu^{2}-1$. Notice that the potential $V_{3}$ is in general complex and energy dependent. For $E$ real and $q>0$ we have

$$
V_{3}=\lambda_{3}\left(R+i V_{\text {abs }}\right)
$$

with

$$
R=\int_{0}^{\infty} \frac{\kappa(k) \mathrm{d} k}{k^{2}-q^{2}} Q_{l}\left(\frac{p^{2}+k^{2}+m_{3}^{2}}{2 p k}\right) Q_{l}\left(\frac{p^{\prime 2}+k^{2}+m_{3}^{2}}{2 p^{\prime} k}\right)
$$




$$
V_{\mathrm{abs}}=\pi \frac{\kappa(q)}{2 q} Q_{l}\left(\frac{p^{2}+q^{2}+m_{3}^{2}}{2 p q}\right) Q_{l}\left(\frac{p^{\prime 2}+q^{2}+m_{3}^{2}}{2 p^{\prime} q}\right) .
$$

In eqs. (5)-(8) we have introduced a phase space factor $\kappa(k)$ to simulate a possible many particle contribution from channel two as is appropriate for the many pion annihilation of $\mathrm{N} \bar{N}$. We have chosen $\kappa(k)$ to be of the form

$$
\kappa(k)=\frac{1}{\sqrt{k^{2}+\mu^{2}}} \begin{cases}1+B\left(k^{2}+E_{t}\right), & \text { for } k^{2}+E_{t}>0 \\ \left(k^{2} /\left(-E_{t}\right)\right)^{n_{1}} & \text { for } k^{2}+E_{t} \leqq 0 .\end{cases}
$$

For the special case that there are only two particles in channel two we have $n_{1}=B=0$. In most of our calculations we shall take $n_{1}=2 \dagger$.

In order to find the solutions of eq. (1) we have adopted the same procedures as described in ref. ${ }^{14}$ ). Since the integral equation (1) is singular for $E>0$, we have used for this case the Kowalski-Noyes technique ${ }^{15}$ ) to reduce these equations to non-singular equations, which are solved numerically by matrix inversion. Having determined the phase shifts $\delta_{l}(E)$ and inelasticity parameter $\eta_{l}(E)$ we may compute the elastic and absorption cross sections from these by

$$
\begin{gathered}
\sigma_{\mathrm{e} 1}=\frac{\pi}{k^{2}}(2 l+1)\left|1-\eta_{l} \mathrm{e}^{2 i \delta l}\right|^{2}, \\
\sigma_{\text {inel }}=\frac{\pi}{k^{2}}(2 l+1)\left(1-\eta_{1}^{2}\right) .
\end{gathered}
$$

To calculate the bound state or resonance poles the zeros were determined of $\operatorname{det}(1-K)$ with $K$ the corresponding kernel of eq. (1) for in general complex energies. This method is applicable for finding the singularities in the first sheet with respect to the threshold of channel one and at the same time in the second sheet with respect to the threshold of channel two. Notice that the analytic continuation of the potential (5) into the second sheet can be carried out by addition of the discontinuity across the cut so that no additional contour deformations are needed to find the behaviour in this region of the $E$-plane. The singularities in the second sheet with respect to the threshold of channel one are in general harder to find, because for that case we need the analytic continuation of the integral equations. In this paper we will not consider this region of the $E$-plane.

In the model described various coupling parameters occur, which have been

$\uparrow$ The average number of pions in the annihilation channels of the $\mathrm{N} \bar{N}$ system is around five ${ }^{13}$ ). The phase space for $n$ relativistic pions behaves as $E^{n-2}$; hence our choice corresponds roughly to $3 \pi$ behaviour. 
allowed to vary over a limited range of values. In order to get some rough idea about the order of magnitude of $\lambda_{i}$ we relate the coupling constants $\lambda_{i}$ to the Lagrangian couplings $g_{i}$. It is given by

$$
\lambda_{i}=\frac{2}{\pi^{2}} \frac{g_{i}^{2}}{4 \pi}, \quad i=1,2
$$

Using the results from one boson exchange models we find that the values of $\lambda_{i}$ are typically of the order of 1 . The region of $\lambda_{3}$ was simply obtained from fitting to the elastic and inelastic low energy $N \bar{N}$ cross sections. Values of the order of one were found. This is consistent with the estimate one finds using the strength of the absorptive part of the Bryan-Phillips potential.

\section{Some properties of the scattering amplitudes}

If we assume that the operator $V^{-1}$ exists, we can formally write the solution of eq. (1) as

$$
t(E)=\left[V^{-1}(E)-G_{0}(E)\right]^{-1},
$$

where $G_{0}$ denotes the free Green function. From this, one readily obtains the following unitarity condition

$$
\operatorname{Im} t(E)=t(E+i \delta)\left[\frac{\operatorname{Im} V}{|V|^{2}}+\pi \delta\left(H_{0}-E\right)\right] t(E-i \delta) .
$$

In our case $\operatorname{Im} V>0$ and consequently eq. (14) has the usual form of the one-channel unitarity equation with some inelasticity. Hence the general solution of eq. (13) can be written in the form ${ }^{12}$ )

$$
t_{l}(E)=\left(\begin{array}{l}
2 \\
\pi
\end{array}\right)^{2} p^{2}\left(p^{2}+1\right)^{\frac{1}{2}} f_{l}(E)
$$

with

$$
f_{l}(E)=\frac{-p^{2 l}}{\alpha_{l}+i \beta_{l}+i p^{2 l+1}}, \quad p=\sqrt{M E}
$$

Here we have explicitly taken into account the threshold behaviour of a given partial wave. The functions $\alpha_{l}$ and $\beta_{l}$ are real analytic functions of $E$ around $E=0$, non-singular at $E=0$ and with the closest singularity at $E=E_{t}<0$. We are interested in the first physical sheet of $E$ and an adjacent part of the second sheet (with respect to $E_{t}$ threshold). Therefore, it is reasonable to draw the cut of $\alpha_{l}, \beta_{l}$ 
as functions of $E$ from $E_{t}$ to $-\infty$, thereby disclosing a part of the second sheet. Note that $\beta_{l}(E)>0$ for $E$ real and $E>E_{t}$, while $\alpha_{l}(E)$ is real for $E>E_{t}$ and otherwise arbitrary. With eq. (16) the elastic and absorption cross sections in a given partial wave $l$ become

$$
\begin{gathered}
\sigma_{\mathrm{el}}^{(l)}=\frac{4 \pi p^{4 l}(2 l+1)}{\alpha_{l}^{2}+\left(\beta_{l}+p^{2 l+1}\right)^{2}}, \\
v \sigma_{\text {inel }}^{(l)}=\frac{4 \pi p^{2 l} \beta_{l}(2 l+1)}{m\left[\alpha_{l}^{2}+\left(\beta_{l}+p^{2 l+1}\right)^{2}\right]} .
\end{gathered}
$$

The analytic properties of $t_{t}(E)$ expressed by eq. (16) and stated above can be inferred from theoriginal eqs. (17),(4) and(5). Indeed from eqs.(4) and (5) we see that $V_{l}\left(p, p^{\prime}, E\right)$ is a real analytic function of $E$, while eq. (1) then provides for the real analyticity of $t_{l}\left(p, p^{\prime}, E\right)$ in the $E$-plane with a cut from $E=E_{t}$ to $\infty$. To get rid of the elastic unitarity cut we can consider the $p$-plane with $p=\sqrt{ } E$ which has a cut from $p_{+}=i \sqrt{\left|E_{t}\right|}$ to $p_{-}=-i \sqrt{\left|E_{t}\right|}$ as is shown in fig. 2. Note that for an odd number of pions inside the annihilation graph the singularity of $V_{3}(E)$ is of a logarithmic type at $E=E_{t}$ and as a consequence the cut drawn in fig. 2 should be connected with the point $E=\infty$ by another cut, which is not shown there. It is more illustrative to draw the cut between $p_{+}$and $p_{-}$as shown in fig. 2 by the dashed line. In this way we disclose the part of the second sheet where the poles move.

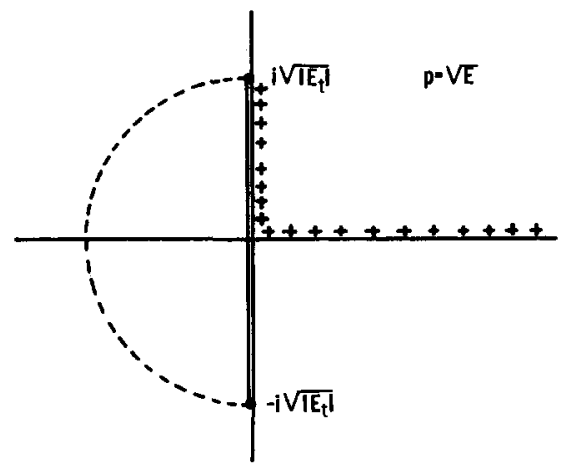

Fig. 2. The complex plane of $p=\sqrt{ } E$ with the cut arising from the presence of the pionic threshold. The physical values of $p$ are marked by crosses. The alternative position of the cut disclosing a part of the second sheet is shown as the dashed line.

We now discuss the number and position of the poles. One way is to use eq. (16) for this. The functions $\alpha_{l}$ and $\beta_{l}$ have dimension [ $\left[p^{2 l+1}\right]$, so that we can write

$$
\alpha_{l}=p_{0}^{2 l+1} \bar{\alpha}_{l}\left(p r_{0}\right) ; \quad \beta_{l}=p_{0}^{2 l+1} \bar{\beta}_{l}\left(p r_{0}\right),
$$


with $\bar{\alpha}_{l}, \bar{\beta}_{l}$ dimensionless and $p_{0}$ an appropriately chosen momentum. The parameter $r_{0}$ stands for the intrinsic parameters of our problem:

(i) the range of interaction related to $m_{1}, m_{2}$ [specifically for $\mathrm{N} \overline{\mathrm{N}}$ with the OBE type interaction $r_{0}$ is of the order of $\left.m_{\sigma_{0}}^{-1} \approx(500 \mathrm{MeV})^{-1}\right]$;

(ii) the inverse distance between the thresholds $\left|p_{+}\right|^{-1}=\left[M\left|E_{t}\right|\right]^{-1}$ (for $\mathrm{N} \overline{\mathrm{N}}$ it is the inverse nuclear mass).

In the case when $p_{0} r_{0} \ll 1$ we can retain one or two terms in the expansion

$$
\begin{aligned}
& \bar{\alpha}_{l}\left(p r_{0}\right)=\bar{\alpha}_{l}^{(0)}+\bar{\alpha}_{l}^{(1)}\left(p r_{0}\right)^{2}+\ldots \\
& \bar{\beta}_{l}\left(p r_{0}\right)=\bar{\beta}_{l}^{(0)}+\bar{\beta}_{l}^{(1)}\left(p r_{0}\right)^{2}+\ldots
\end{aligned}
$$

With this the denominator of eq. (16) is a polynomial of degree $2 l+1$ with $2 l+1$ roots. Hence we have $2 l+1$ poles in the $N \bar{N}$ amplitude, all of them being approximately at a distance $p_{0}$ from the origin. The value $p_{0}$ can be estimated from the phenomenological description of the experimental data with the aid of eqs. (17) and (18). For the $\mathrm{NN}$ case $p_{0} \lesssim 0.3 \mathrm{GeV} / c$ for $l=0,1$.

Let us now turn to some general analytic properties of the scattering matrix. The poles in the $p$-plane should be symmetric with respect to the imaginary axis. This property can be seen from eq. (16) as follows. From the $K$-matrix formalism one immediately finds, that $\beta_{l}(p)$ is proportional to $q=\sqrt{E-E_{t}}$,

$$
\beta_{l}=q \phi_{l}(E)
$$

with $\phi_{l}(E)$ real analytic in $E$ and even under exchange of $p \rightarrow-p$. Also for $E$ on the right-hand side of the $\left(p_{+}, p_{-}\right)$cut (see fig. 2) one has $\phi_{l}>0$. With these properties of $\beta_{l}(p)$ and $\alpha_{l}\left(-p^{*}\right)=\alpha_{l}^{*}(p)$ we immediately find by analytic continuation of eq. (21) through the $\left(p_{+}, p_{-}\right)$cut that if eq. (16) has a pole at $p=a$ then it has also a pole at $p=-a^{*}$. Another consequence of eq. (21) is that an apparent difficulty of eq. (16) can be resolved. For simplicity, let us consider the case of $l=0$. If we keep $\alpha$ and $\beta$ constant, then the pole of eq. (16) would be given by $p=-\beta_{0}+i \alpha_{0}$, which seemingly lies in the first sheet of the $p$-plane. However taking eq. (21) into account we see that in view of $\operatorname{Re} p=-\beta_{0}<0$, the pole is actually in the second sheet with respect to the $\left(p_{+}, p_{-}\right)$cut. With regard to the trajectory of a pole as a function of some parameter, we know that since $\beta_{l}(p)$ is real everywhere on the cut $\left(p_{+}, p_{-}\right)$and purely imaginary on the imaginary axis of $p$ outside this cut, that no trajectory can cross the cut $\left(p_{+}, p_{-}\right)$. Indeed, at the point of crossing on the cut $\alpha_{l}(p)$ is real, so is $i p^{2 l+1}$, while $i \beta_{l}(p)$ is imaginary and as a consequence eq. (16) cannot have a pole.

It is sometimes useful to represent the elastic amplitude in an Argand plot. Keeping only the first two terms in the expression of $\alpha_{l}$ and $\beta_{l}$ as in eq. (20), we have, using the analytic representation (16), for the real and imaginary parts of the $N \bar{N}$ scattering 
amplitude,

$$
\begin{aligned}
& \operatorname{Re}\left(p f_{l}\right)=\frac{-p^{2 l+1}\left(\alpha_{0}+\alpha_{1} p^{2}\right)}{\left(\alpha_{0}+\alpha_{1} p^{2}\right)^{2}+\left(\beta_{0}+\beta_{1} p^{2}+p^{2 l+1}\right)^{2}}, \\
& \operatorname{Im}\left(p f_{l}\right)=\frac{p^{2 l+1}\left(\beta_{0}+\beta_{1} p^{2}+p^{2 l+1}\right)}{\left(\alpha_{0}+\alpha_{1} p^{2}\right)^{2}+\left(\beta_{0}+\beta_{1} p^{2}+p^{2 l+1}\right)^{2}} .
\end{aligned}
$$

Qualitatively four different types of Argand plots are possible, depending on the signs of $\alpha_{0}, \alpha_{1}$. These are shown for the case of s-waves in fig. 3. Only one of the Argand plots namely that for $\alpha_{0}<0$ and $\alpha_{1}>0$ resembles that of the usual BreitWigner resonance. In all four cases we have $2 l+1$ poles in the vicinity of the threshold.

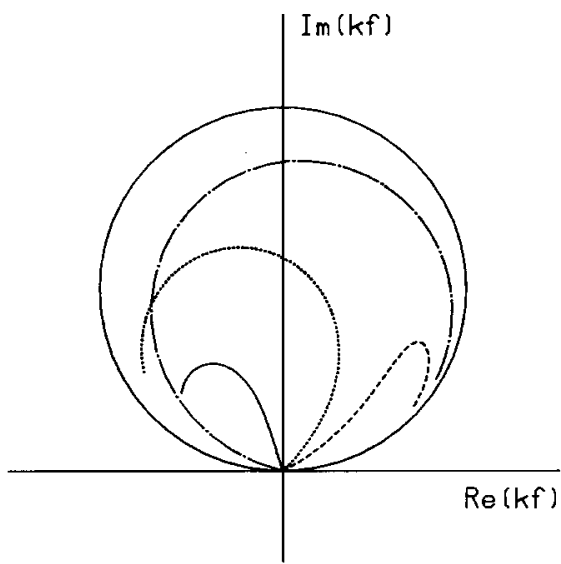

Fig. 3. The Argand plots corresponding to eqs. (22) and (23) with $L=0$ and different choices of $\alpha_{0}, \alpha_{1}$. The solid line is for $\alpha_{0}, \alpha_{1}>0$, the dashed line is for $\alpha_{0}, \alpha_{1}<0$, the dotted line is for $\alpha_{0}<0, \alpha_{1}>0$ and the dash-dot line is for $\alpha_{0}>0, \alpha_{1}<0$.

\section{Results and discussion}

In a realistic calculation using interactions arising from exchange of more bosons, the $\mathbf{N} \bar{N}$ force will in general be state dependent. In a simple model as considered here this state dependence has been simulated by allowing the coupling parameters $\lambda_{i}$ to be dependent on the partial wave studied. At the energies considered and for the coupling constants of the order of one the contributions from the partial waves $l \geqq 2$ were found to be small. As a consequence we have confined ourselves to the study of the s- and p-waves only, although in a more refined calculation the higher partial waves as well as the spin-isospin effects should be taken into account. In this paper we are mainly interested in the possible effects which can occur qualitatively if an annihilation channel is included. In particular, the motion of bound state 
singularities as a function of $\lambda_{3}$ and the various effects on observables of the $N \bar{N}$ system have been studied.

We have solved eq. (1) numerically fixing from the beginning the parameters $m_{1}, m_{2}$ and $m_{3}$ to be 770,1500 and $939 \mathrm{MeV}$, respectively. The parameter $n_{1}$ was taken to be two in order to simulate the four-body phase space. In some numerical runs $n_{1}$ was taken to be 0 and

$$
\kappa(k)=\frac{1+B \sqrt{k^{2}+E_{t}}}{\sqrt{k^{2}+\mu^{2}}}, \quad k^{2}+E_{t} \geqq 0
$$

instead of eq. (9). No qualitative different results were thereby found. Two different choices for $B$ were used: (i) $B=0$ which is specific for a two-body phase space and (ii) $B=2$ which is characteristic for $\operatorname{Im} V_{3}(E=0) \approx \operatorname{Re} V_{3}(E=0)$. The latter condition was shown in ref. ${ }^{8}$ ) to be optimal for reproducing $\sigma_{\text {el }}(E)$ and $v \sigma_{\text {inel }}(E)$. The parameter $\mu$ was taken to be twice the pion mass. This is in accordance with our notion that experimentally the largest contribution to $\mathrm{N} \overline{\mathrm{N}}$ annihilation comes from final states with four to seven pions. Although in a more realistic calculation variations of the adjustable parameter $\mu$ should be considered, no qualitatively different results are expected from what we have found here.

The parameters $\lambda_{1}, \lambda_{2}$ and $\lambda_{3}$ were varied to reproduce the experimental values of $\sigma_{\mathrm{el}}(E)$ and $v \sigma_{\text {inel }}(E)$. Having determined $\lambda_{i}$, we then studied the trajectories of the poles of the $N \bar{N}$ amplitude in the complex $E$-plane as a function of the coupling

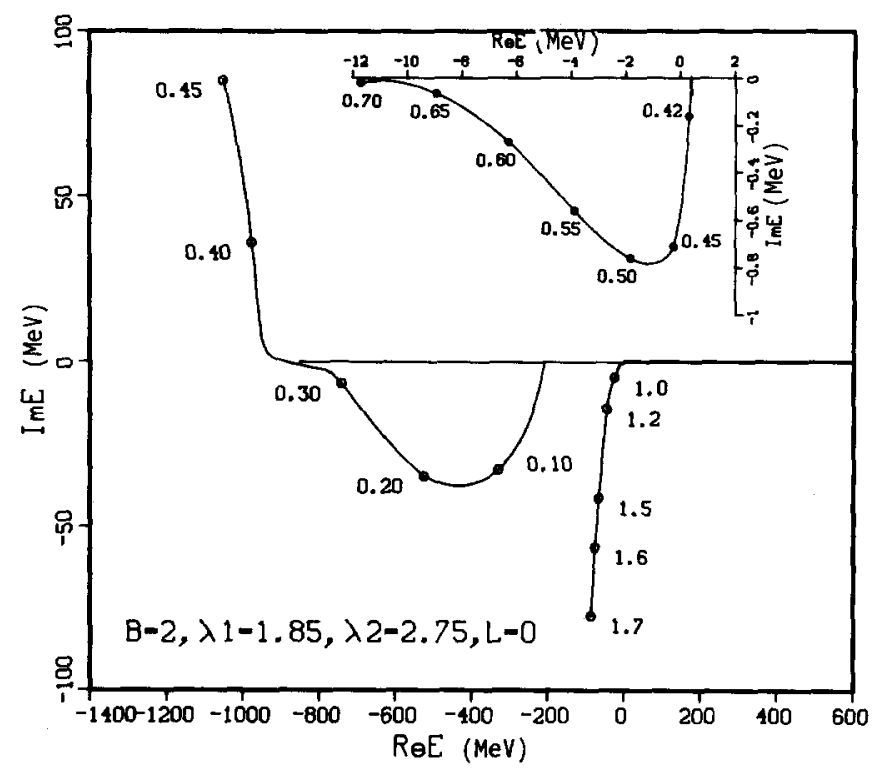

Fig. 4. The trajectories of the quasi-nuclear pole (Q-pole; left) and the coupled channel pole (CC pole; right) in the complex energy plane as a function of $\lambda_{3}$. Numbers at the curve are the values of $\lambda_{3}$. 


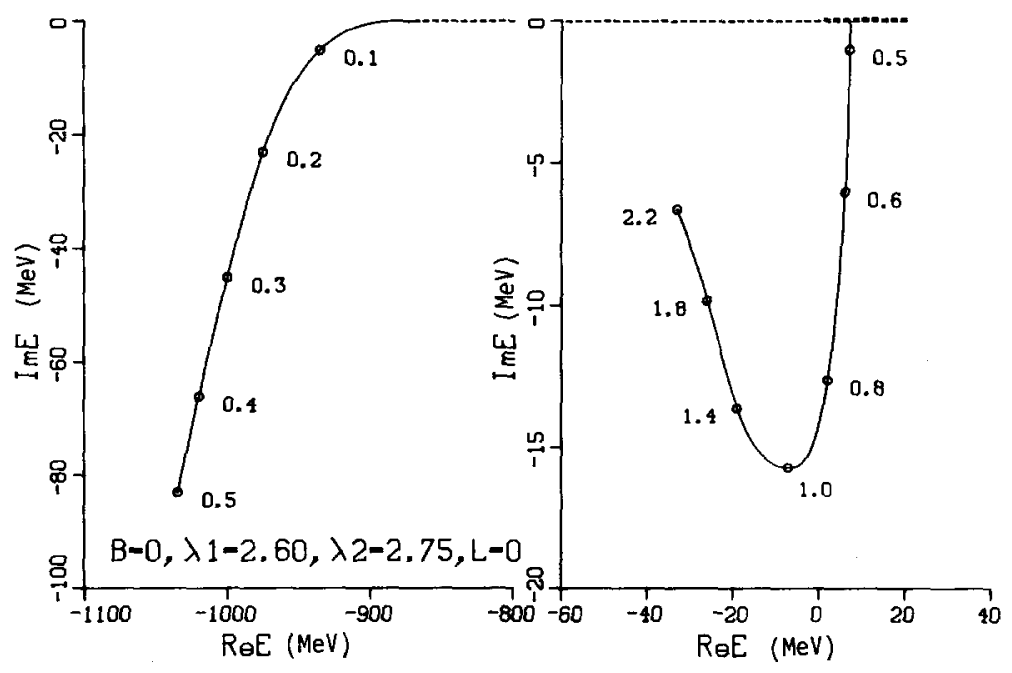

Fig. 5. The same as in fig. 4.

constant $\lambda_{3}$. The result is shown in fig. 4 for $l=0$. From this we see that on increasing $\lambda_{3}$ a second singularity appears near $E=0$. It initially moves downward, turn back to the real $E$-axis (see inset in fig. 4) and after that moves again downward, with a relatively small shift in the real part of the energy. Since in the explicit calculation of the $N \bar{N}$ spectrum without annihilation some low lying states were also found ${ }^{3}$ ) we considered the effect of annihilation for a situation where the bound state pole is at $-870 \mathrm{MeV}$ when $\lambda_{3}=0$ (see fig. 5). The following features of the trajectories shown in figs. 4 and 5 are interesting to note. For both sets of parameters two types of trajectories arise. One originates from a bound state when $\lambda_{3}=0$ and is of a quasi-nuclear nature (Q-pole). With increasing $\lambda_{3}$ it shifts to the left which corresponds to more binding. Needless to say, the description with quasi-potential models of these bound states with large binding energies may not be reliable any more and that other effects like non-kinematical relativistic effects may become very important. Another trajectory emerges from the second sheet with respect to $\mathrm{NN}$ threshold, crossing the real axis at $1-7 \mathrm{MeV}$ to the right of the threshold. The pole is close to threshold for "physical" $\lambda_{3}$ values, i.e. the $\lambda_{3}$ values giving cross sections which are in reasonable agreement with the experimental ones. Such a pole we will call a coupled channel pole (CC pole). Furthermore, the magnitude of the energy shift of a Q-pole due to annihilation is $\approx 100 \mathrm{MeV}$ for $B=0$ and $\approx 600 \mathrm{MeV}$ for $B=2$, in qualitative agreement with the $N / D$ calculations. For even even stronger $\lambda_{3}\left(\lambda_{3} \geqq 6\right)$ than shown in these figures another CC pole appears near $E=0$ in the first $N \bar{N}$ sheet and which moves to the right with increasing $\lambda_{3}$, reaching a position $E \approx 200-i 40 \mathrm{MeV}$ for $\lambda_{3} \approx 11$. In this connection it is worthwhile noting that there is a rather unexpected behaviour of $v \sigma_{\text {inel }}(E)$ with $E$ fixed 


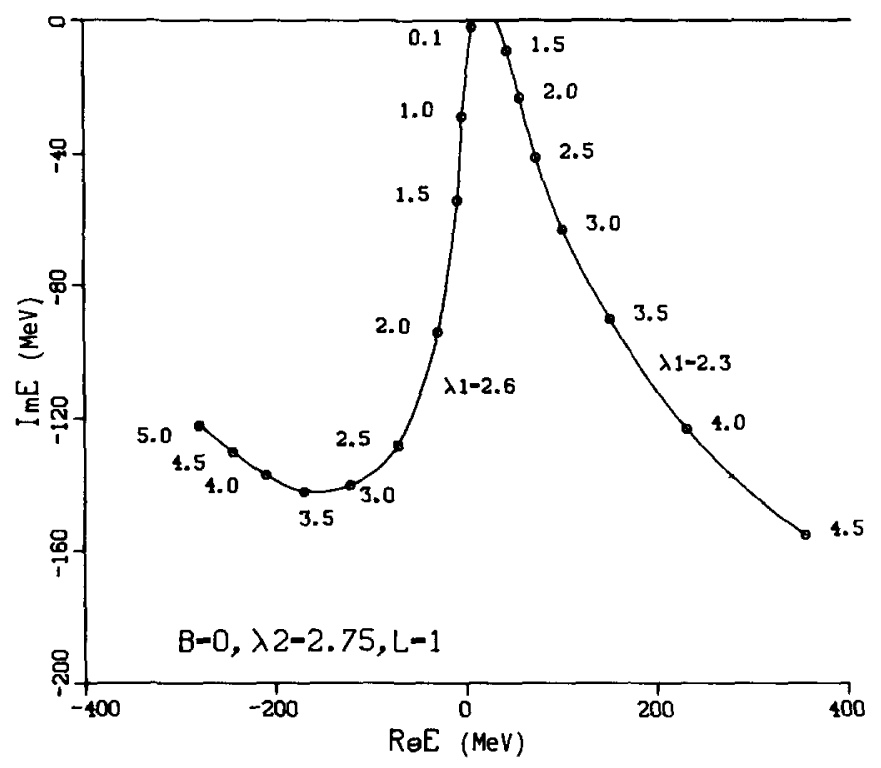

Fig. 6. The trajectories of the $\mathrm{CC}$ poles for various sets of parameters.

and $\lambda_{3} \rightarrow \infty$, namely $v \sigma_{\text {inel }}(E)$ oscillates close to but below the unitary limit and only at very high $\lambda_{3}$ starts to decrease. This might be an indication that more CC poles are emerging close to threshold into the first sheet with increasing $\lambda_{3}$.

We now turn to the $l=1$ case. Here only one pole is found for the set of parameters

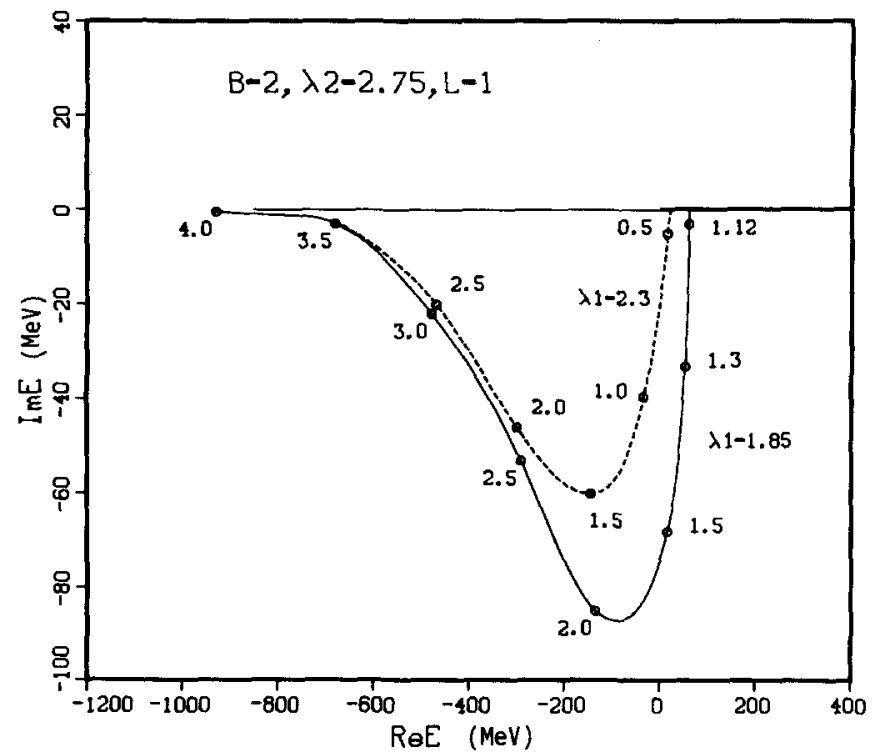

Fig. 7. The same as in fig. 6 . 
used for $l=0$. The coupling constant $\lambda_{1}$ is not strong enough to produce a p-wave bound state for $\lambda_{3}=0$. Since the $\mathrm{NN}$ force is expected to depend on the partial wave considered we have changed the parameters $\lambda_{1}, \lambda_{3}$ to get reasonable cross sections for $l=1$. In figs. 6 and 7 the trajectories are shown for different sets of parameters. The physical values for $l=1$ cross sections $\sigma_{\mathrm{el}}$ and $v \sigma_{\text {inel }}$ are given for the two sets of parameters by $B=0, \lambda_{1}=2.3, \lambda_{3}=1.3$ and $B=2, \lambda_{1}=2.3, \lambda_{3}=0.7$. Notice that the $\lambda_{3}$ values are several times larger than for the $l=0$ case. For the above sets of parameters we find that the positions of the $\mathrm{CC}$ poles are given by $E_{\mathrm{p}}=37.2-4.83 i \mathrm{MeV}$ and $E_{\mathrm{p}}=5.52-16.8 i \mathrm{MeV}$ respectively. Both poles are in the first $N \bar{N}$ sheet and second pionic sheet. From fig. 6 we see that it is possible by a change in $\lambda_{1}$ to get a situation where the annihilation becomes effectively repulsive. To get an idea whether there is a resonance of the Breit-Wigner type (BW) present in the second $N \bar{N}$ sheet, we calculated the phase shift $\delta$ as a function of energy for the case that $\lambda_{3}=0$. This is shown in fig. 8 . From this we see that for the physical value of $\lambda_{1}=2.3$ a resonance develops at around $45 \mathrm{MeV}$, while for a somewhat smaller $\lambda_{1}=1.85$ the phase shift is less than $70^{\circ}$ up to $250 \mathrm{MeV}$.

The annihilation cross sections for two sets of parameters with $B=0$ and $B=2$ are given in figs. 9 and 10 together with the lowest in energy experimental points. The $l=1$ cross sections show broad bumps at around $15-20 \mathrm{MeV}$ while the $l=0$ contribution is sharply peaked at the threshold. Both facts are in accordance with the presence of $\mathrm{CC}$ poles in $l=0,1$. The contribution of the $l=2$ partial wave is small below $30 \mathrm{MeV}$ and is not shown here. The elastic cross sections for the same two sets of parameters are shown in figs. 11 and 12. The most striking effect is the

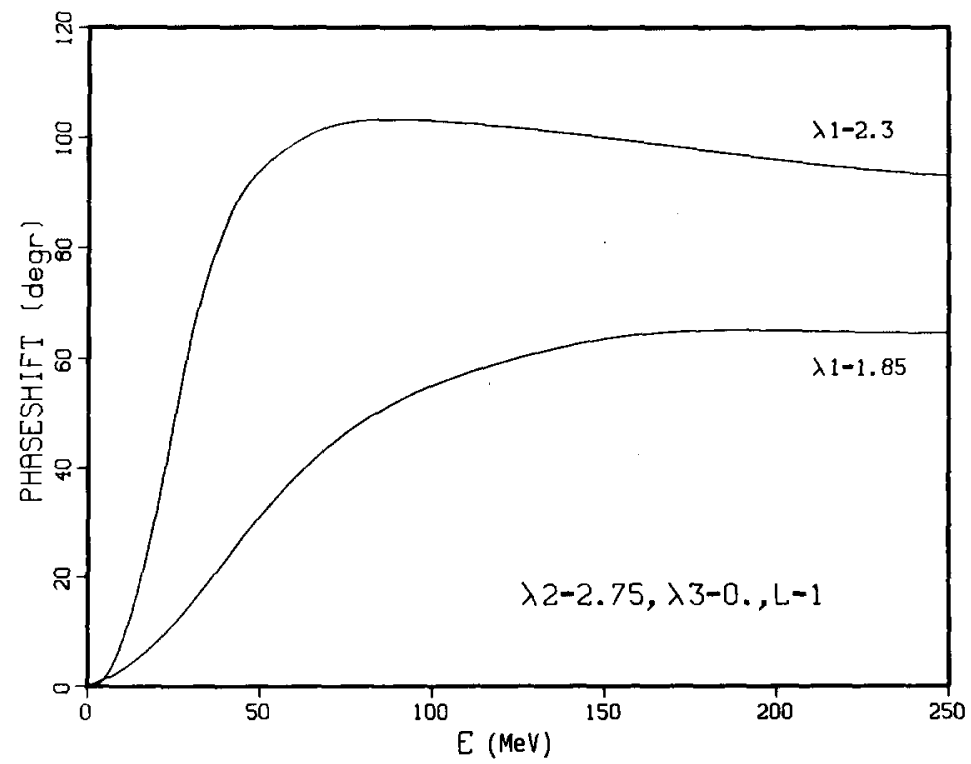

Fig. 8. The phase shift $\delta$ as a function of energy. 


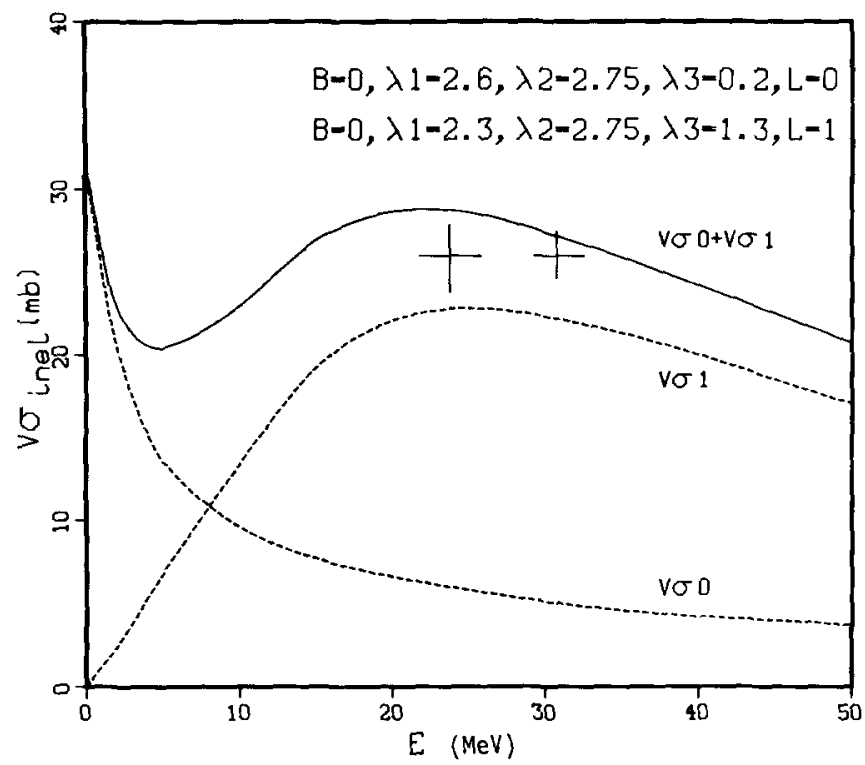

Fig. 9. The annihilation cross sections $v \sigma_{\text {inel }}$ as a function of energy for $l=0$ and $l=1$ and their sum. For comparison the experimental points are shown.

peak at the threshold in the total elastic cross section, coming from the $l=0$ contribution with a width of the order of $10 \mathrm{MeV}$. The total elastic cross section rises from $\approx 100 \mathrm{mb}$ at $9 \mathrm{MeV}$ (where the lowest experimental point is) to $260 \mathrm{mb}(B=2)$

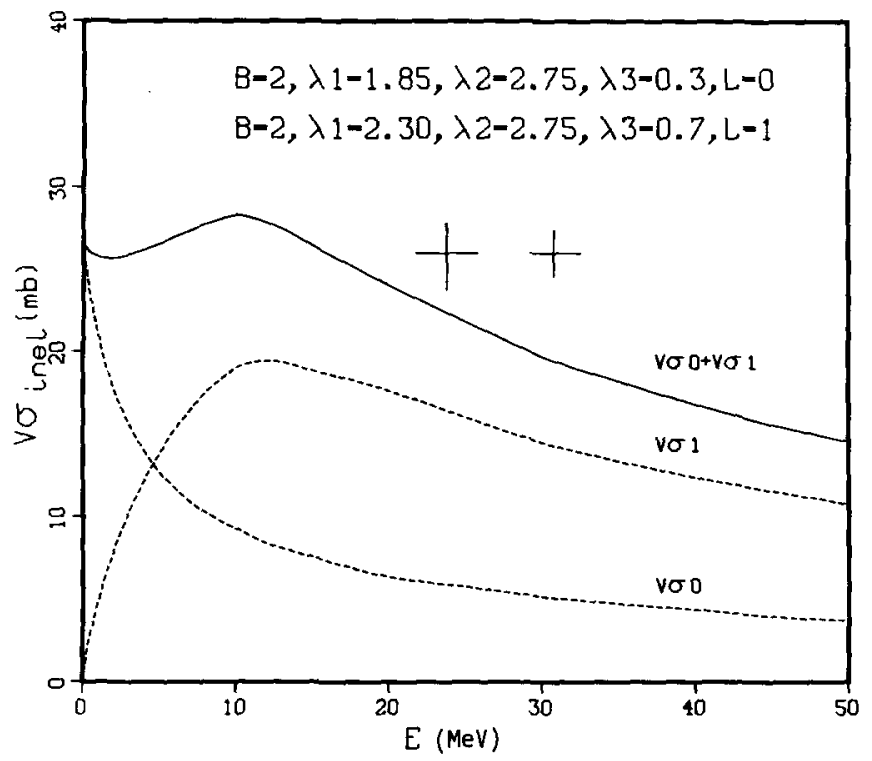

Fig. 10. The same as in fig. 9 . 


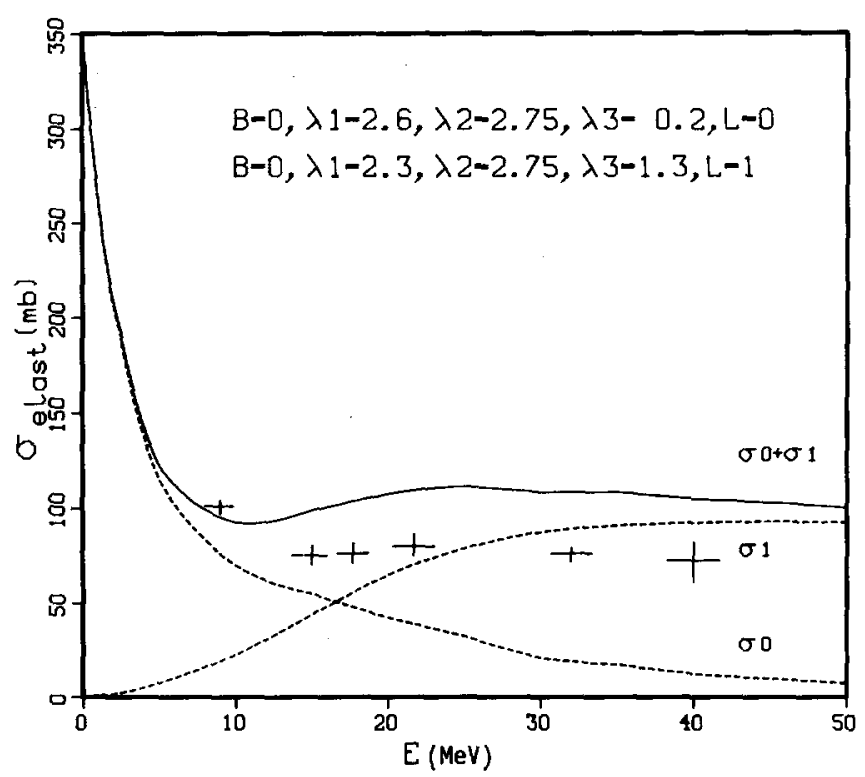

Fig. 11. The elastic cross sections $\sigma_{\mathrm{el}}$ as a function of energy for $l=0$ and $l=1$ and their sum. For comparison the experimental points are shown.

or $350 \mathrm{mb}(B=0)$ at $E=0.1 \mathrm{MeV}$. This effect is wholely due to the presence of the nearby $C C$ poles in $l=0$ and can be simply described with eq. (17). Note that a similar effect is present in the calculations with the Bryan-Phillips potential, where

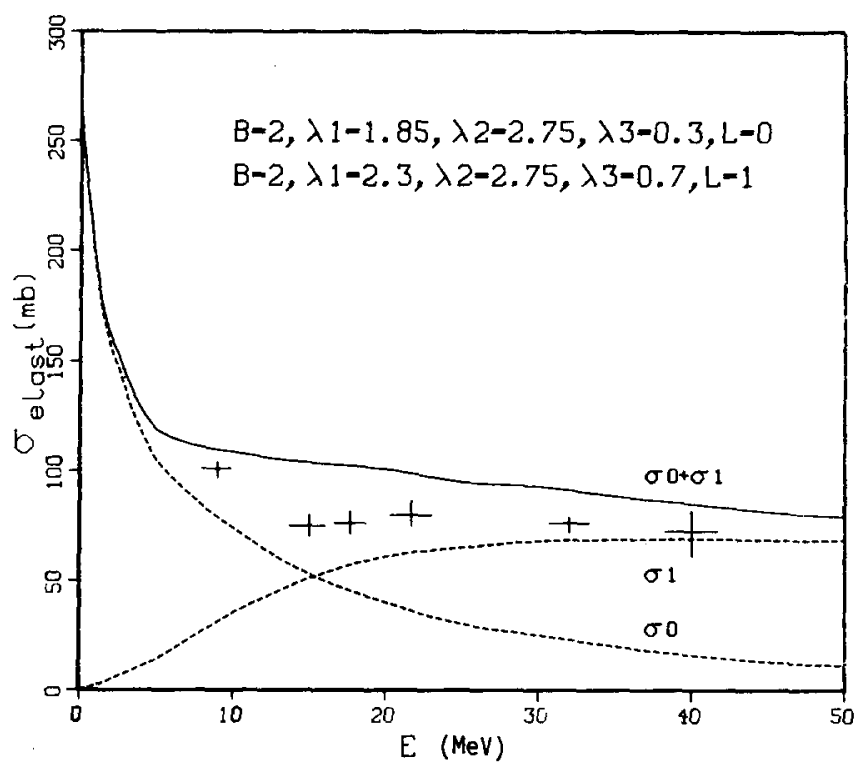

Fig. 12. The same as in fig. 11 . 
the total cross section is $180 \mathrm{mb}$ at zero energy and $90 \mathrm{mb}$ at $E=10 \mathrm{MeV}$. Beyond that peak the sum of $l=0,1$ cross sections is rather flat in accordance with the experimental data.

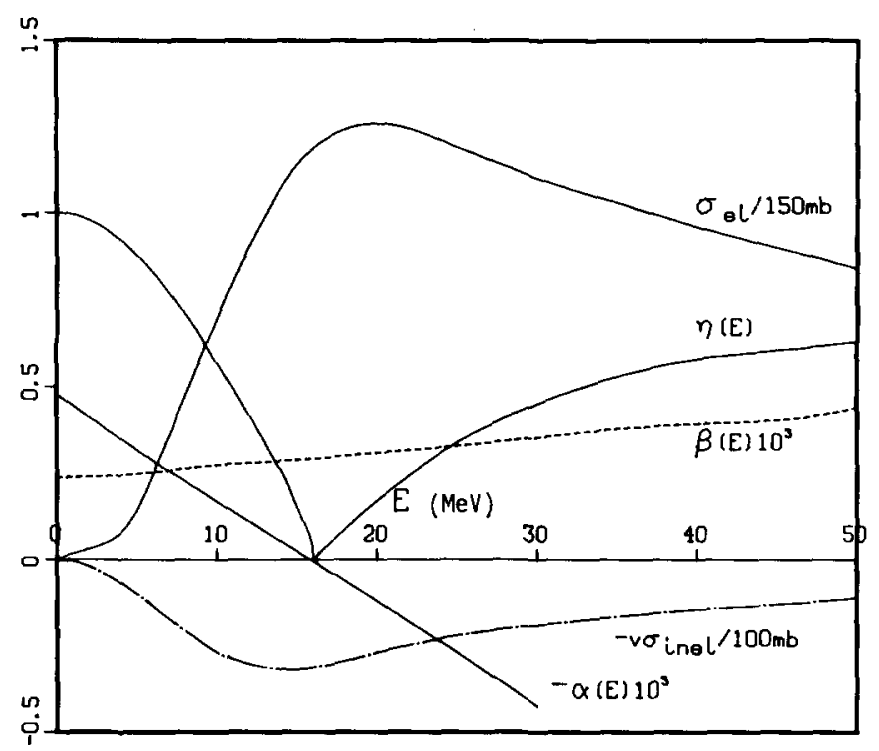

Fig. 13. The elastic cross section $\sigma_{\mathrm{el}}$ (in units of $150 \mathrm{mb}$ ); the annihilation cross section $v \sigma_{\text {inel }}$ (in units of $100 \mathrm{mb}$ ), the inelasticity parameter $\eta(E)$ and the functions $\alpha(E), \beta(E)$ obtained by fitting the cross sections with eqs. (17) and (18).

A comparison of our annihilation cross sections $v \sigma_{\text {inel }}$ with the upper unitary limit $\pi(2 l+1) / k^{2}$ shows that we are in the regime very close to a black sphere. In our case the occurrence of a black sphere is due to the CC pole. Specifically from eqs. (17) and (18) we deduce that the black sphere situation is realized when $\beta_{I} \gg\left|\alpha_{l}\right|$ in the energy interval $p^{2 l+1} \approx \beta_{l}$. To show this effect explicitly we have drawn in fig. 13 the $l=1$ case for the set of parameters with $\lambda_{3}$ such that the $C C$ pole just crosses the real axis ( $\alpha_{l}$ is small). From this figure we see that the inelasticity $\eta(E)$ passes through zero exactly at the point where $\alpha(E)$ crosses the real axis while $\sigma_{\mathrm{el}}$ has a bump there. The vanishing of $\eta(E)$ at a given point and continuity arguments suggest the possible origin of the $\mathrm{CC}$ poles we have found. In view of the symmetry $p \rightarrow-p^{*}$ discussed in the previous section and the presence of a $\mathrm{BW}$ resonance, we know that there is a singularity for $\lambda_{3}=0$ in the upper part of the second $N \bar{N}$ sheet and which is conjugated to the BW pole. Although this singularity is normally far away from the physical region, due to the switching on of the annihilation channel, the singularity is pulled towards the real axis, crossing it near threshold and emerging then as a CC pole in the second pionic sheet. The inelasticity parameter $\eta(E)$ becomes vanishing small at the point where the $\mathrm{CC}$ pole appears on the real axis. The energy 
where the NN system is completely black is $16 \mathrm{MeV}$, which is indeed not far from the position of the $\mathrm{BW}$ resonance shown in fig. 8. Another interesting observation is the validity of the effective range description in the energy region considered here. The coefficients $\alpha$ and $\beta$ from eq. (16) were determined at a given energy from the calculated values of $\eta$ and $\delta$. The results are shown in fig. 13 for the $l=1$ case. An almost linear dependence of $\alpha$ and $\beta$ on $E$ is found. From this we may infer that although the amplitude itself varies very strongly as a function of the energy, the effective range parameterisation of $\alpha$ and $\beta$ given by eq. (20) is in reasonable accordance with the calculated results. From this we may conclude that the range parameter $r_{0}$ is indeed sufficiently small that the higher order terms in the expansion do not contribute significantly.

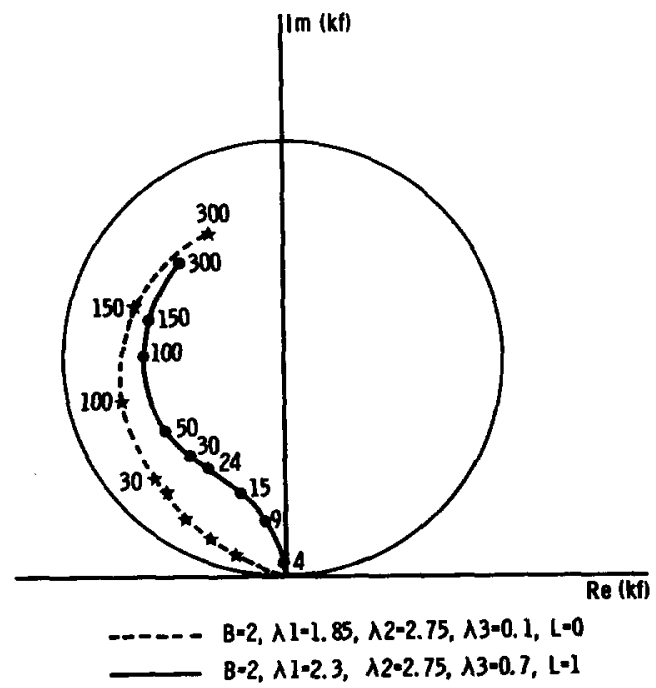

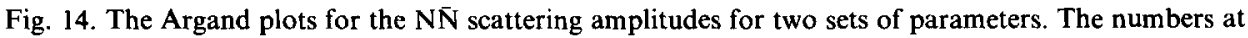
the curves give the corresponding c.m. energies.

Finally we turn to the discussion of the Argand plots which are shown in the next two figures for some sets of parameters. The loops in fig. 14 are similar to the one in fig. 3 with $\alpha_{0}>0, \alpha_{1}<0$. In fig. 15 three curves are shown for $\lambda_{3}=0, \lambda_{3}=0.3$ and $\lambda_{3}=1.3$ to illustrate the increasing effect of annihilation. The purely elastic loop for $\lambda_{3}=0$ describes a BW resonance at $45 \mathrm{MeV}$, which is in accordance with fig. 8. Increasing $\lambda_{3}$ to 0.3 yields a slightly deformed curve, while for the physical value of $\lambda_{3}=1.3$ we find one $S$-shaped curve where one hardly recognizes a BW resonance contribution. Still in this case we know that there is a $\mathrm{CC}$ pole at $E_{\mathrm{p}}=37.2-4.83 i \mathrm{MeV}$ as discussed above. From our discussion in sect. 3 we also know that for $l=1$ there should be three poles near the threshold. To find their position we have analyzed the phase parameters $\eta(E)$ and $\delta(E)$ with functions $\alpha(E)$ 


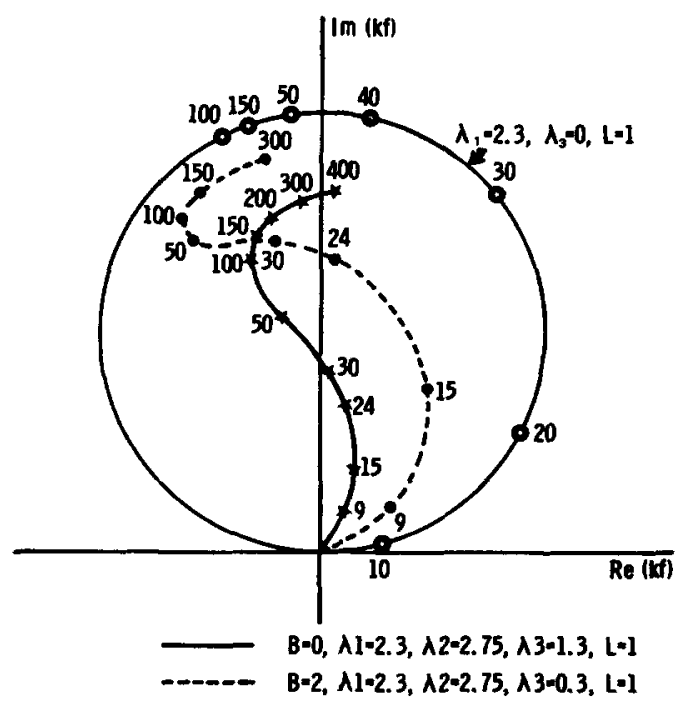

Fig. 15. The same as in fig. 14, but with three sets of parameters.

and $\beta(E)$ linear in $E$ and solved the resulting cubic equations for the pole positions. They are given by

$$
\begin{array}{ll}
E_{1}=4.01-23.55 i \mathrm{MeV} & \text { (second } \mathrm{N} \bar{N} \text { sheet), } \\
E_{2}=-61.36+3.32 i \mathrm{MeV} & \text { (second } \mathrm{N} \overline{\mathrm{N}} \text { sheet), } \\
E_{3}=39.3-2.15 i \mathrm{MeV} & \text { (first } \mathrm{N} \overline{\mathrm{N}} \text { sheet). }
\end{array}
$$

All these poles are in the second pionic sheet. Note the presence of the BW resonance $E_{1}$ with a width of $\Gamma=47 \mathrm{MeV}$ which probably has shifted due to the annihilation from its original position at $45 \mathrm{MeV}$. The third pole at $E_{3}$ is a CC pole which should be identified with the one calculated at $E=37.2-4.83 i \mathrm{MeV}$. The difference gives an idea of the accuracy of the linear approximation for $\alpha(E)$ and $\beta(E)$.

It is remarkable that our curve with $\lambda_{3}=1.3$ in fig. 15 looks very similar to that of Myhrer and Gersten, calculated for the $P_{33}$ wave using the Bryan-Phillips potential. From the deformed shape of this curve Myhrer and Gersten concluded that the resonances in that wave are killed by annihilation. However as we have seen the S-shaped loop is a result of a combined effect of three poles. The same is true for the Myhrer-Gersten case. Using the same procedure as above we obtain for the $W_{0}=2 \mathrm{GeV}$ case

$$
\begin{array}{ll}
E_{1}=4.71-10.36 i \mathrm{MeV} & \text { (second } \mathrm{N} \bar{N} \text { sheet) } \\
E_{2}=-54.1-20.61 i \mathrm{MeV} & \text { (first } \mathrm{N} \bar{N} \text { sheet) } \\
E_{3}=18.58-5.2 i \mathrm{MeV} & \text { (first } \mathrm{N} \bar{N} \text { sheet). }
\end{array}
$$



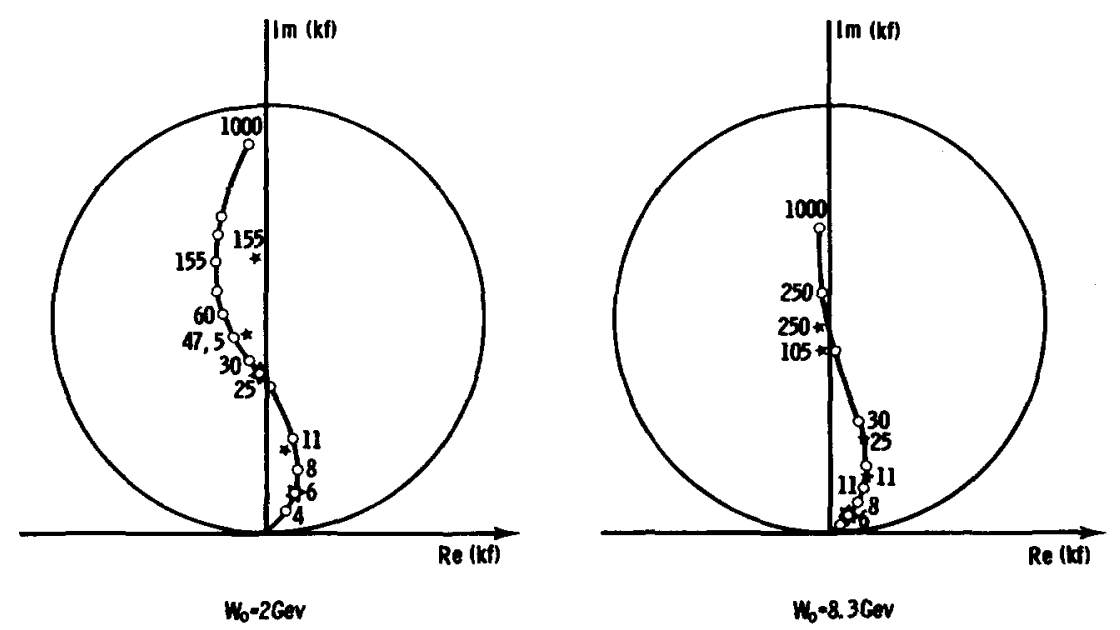

Fig. 16. The Argand plots for the $\mathrm{N} N$ scattering amplitudes for the $W_{0}=2$ and $8.3 \mathrm{GeV}$ case: stars are points calculated by Myhrer and Gersten; circles, a fitting using eqs. (22) and (23). The values on the curves are the corresponding c.m. energies.

Again $E_{3}$ corresponds to the $\mathrm{CC}$ pole. Notice that the poles are quite near the threshold and real axis in contrast to the conclusions of Myhrer and Gersten. Similarly we have analyzed the $W_{0}=8.3 \mathrm{GeV}$ case. Our fits with linear functions in $E$ of $\alpha$ and $\beta$ are shown in fig. 16. The poles for the $W_{0}=8.3 \mathrm{GeV}$ case are found to be

$$
\begin{array}{ll}
E_{1}=0.683-12.54 i \mathrm{MeV} & \text { (second } \mathrm{N} \bar{N} \text { sheet) } \\
E_{2}=-6.68-17.04 i \mathrm{MeV} & \text { (first } \mathrm{N} \bar{N} \text { sheet) } \\
E_{3}=186.9-4.66 i \mathrm{MeV} & \text { (first } \mathrm{N} \bar{N} \text { sheet) }
\end{array}
$$

From the above examples we see that the width of the Q-pole $\left(E_{1}\right)$ can be rather small, but that its effect on the Argand plot is masked by the contribution of the CC pole $\left(E_{3}\right)$.

Most part of this work was done while one of the authors (Yu. S.) was on a twomonth visit to the Netherlands in the framework of an agreement between the atomic energy commission of USSR and the Reactor Center in the Netherlands. This author would like to thank the theoretical groups of the University of Utrecht and of the Free University of Amsterdam for their warm hospitability. He also acknowledges the cooperation of all the authorities involved. We are grateful to M. I. Polikarpov for help in the computation of the two last examples. 


\section{References}

1) L. Montanet, Fifth Int. Conf. on experimental meson spectroscopy, Boston, April 1977, Cern (EP) Phys. 77-22

2) G. C. Rossi and G. Veneziano, Nucl. Phys. B23 (1977) 507

3) I. S. Shapiro, Sov. Phys. Usp. 16 (1973) 173;

L. N. Bogdanova, O. D. Dalkarov and I. S. Shapiro, Ann. of Phys. 84 (1974) 261 ;

J. M. Richard et al., Phys. Lett. 64B (1976) 121;

C. B. Dover and M. Goldhaber, Phys. Rev. D15 (1977) 1997;

J. A. Tjon, Phys. Rev. D18 (1978) 2565;

G. Schierholz and S. Wagner, Nucl. Phys. B32 (1971) 306

4) 1. S. Shapiro, Phys. Reports 35C (1978) 129

5) F. Myhrer and A. Gersten, Nuovo Cim. 37A (1977) 21

6) F. Myhrer and A. W. Thomas, Phys. Lett. 64B (1976) 59

7) O. D. Dalkarov and F. Myhrer, Nuovo Cim. 40A (1977) 152

8) A. M. Badalyan, M. I. Polikarpov and Yu. A. Simonov, Phys. Lett. 76B (1978) 388

9) B. R. Karlsson and B. Kerbikov, Nucl. Phys. B141 (1978) 241

10) R. A. Bryan and R. J. N. Phillips, Nucl. Phys. B5 (1968) 201

11) A. A. Logunov and A. N. Tavkhlidze, Nuovo Cim. 29 (1963) 380;

R. Blankenbecler and R. Sugar, Phys. Rev. 142 (1966) 1051

12) R. Dalitz and S. F. Tuan, Ann. of Phys. 3 (1960) 307;

J. D. Jackson et al., Nuovo Cim. 9 (1958) 834

13) J. E. Ekstrom et al., A compilation - NN and Nd interactions, LBL-58 (1972)

14) M. J. Levine, J. Wright and J. A. Tjon, Phys. Rev. 154 (1967) 1433

15) K. L. Kowalski, Phys. Rev. Lett. 15 (1965) 798;

H. P. Noyes, Phys. Rev. Lett. 15 (1965) 538 